\title{
La musique au Musée national des Arts et Traditions populaires et au futur Musée des civilisations de l'Europe et de la Méditerranée
}

Florence Gétreau et Michel Colardelle

\section{(2) OpenEdition \\ Journals}

Édition électronique

URL : http://journals.openedition.org/ethnomusicologie/574

ISSN : 2235-7688

\section{Éditeur}

ADEM - Ateliers d'ethnomusicologie

Édition imprimée

Date de publication : 1 novembre 2003

Pagination : 43-58

ISBN : 978-2-8257-0863-7

ISSN : $1662-372 X$

\section{Référence électronique}

Florence Gétreau et Michel Colardelle, « La musique au Musée national des Arts et Traditions populaires et au futur Musée des civilisations de l'Europe et de la Méditerranée », Cahiers

d'ethnomusicologie [En ligne], 16 | 2003, mis en ligne le 16 janvier 2012, consulté le 20 avril 2019. URL : http://journals.openedition.org/ethnomusicologie/574 


\title{
La musique au Musée national des Arts et Traditions populaires et au futur Musée des civilisations de l'Europe et de la Méditerranée
}

\author{
Florence Gétreau et Michel Colardelle
}

1 Le rôle patrimonial du Musée national des Arts et Traditions populaires (MNATP) a toujours été, depuis sa fondation en 1937 et son ouverture au bois de Boulogne en 1972, spécifique au sein des musées de France : il s'agissait de constituer une collection la plus représentative possible des pratiques sociales et culturelles de la France dans son contexte européen, durant une période (l'époque moderne jusqu'à la « fin des paysans » au lendemain de la Seconde Guerre mondiale) choisie en fonction de la cohérence historique et culturelle qu'on lui prêtait. Les objets matériels collectés, conservés et montrés, fruits d'élaborations culturelles à tous les sens du terme, étaient considérés comme les signes d'un langage symbolique dont la portée dépasse la seule fonctionnalité. A ce titre, ils étaient les témoins des différentes sociétés qui se sont succédées dans le temps ou se juxtaposent dans l'espace, en s'attachant spécifiquement aux strates populaires et, par conséquent, en privilégiant la campagne, monde agricole et artisanal, considéré comme un conservatoire, temporaire mais en voie à son tour d'évolution, de traditions déjà disparues en contexte urbain. Les objets ne pouvaient donc prendre tout leur sens qu'accompagnés des résultats d'une recherche contextuelle qui les faisait dépasser leur valeur apparente, technique ou esthétique. Recherche et présentation ou diffusion s'inscrivaient dans une perspective synthétique nationale, négligeant ou minorant à la fois le fait régional et le cosmopolitisme urbain. Il ne s'agissait donc pas vraiment d'un musée généraliste, mais plutôt d'un musée thématique. Le laboratoire de recherche spécialisé qu'il constituait avec le CNRS, le Centre d'ethnologie française (EF, aujourd'hui UMR 306 du CNRS), dont les travaux furent d'abord étroitement liés à cette politique, avait progressivement diversifié ses thématiques en s'ouvrant à la fois sur l'Europe, sur la sociologie et sur la ville, sans que le musée, dans ses acquisitions et 
surtout dans l'image publique qu'en donnaient ses galeries permanentes, suive cette transformation. Les faits socio-culturels étaient étudiés dans leur complexité, dans leurs rapports réciproques, dans leur contexte, en mobilisant toutes les disciplines utiles, sans exclusive - les grandes enquêtes telles que celles de Sologne ou d'Aubrac, dont le musée avait la responsabilité, étaient largement pluridisciplinaires -, avec toutefois, s'agissant de «traditions populaires", une dominante ethnologique et sociologique accompagnant le caractère ethnographique des collections.

2 Par ailleurs les « collections immatérielles ", traditions orales, comportements collectifs, rites, que l'enquête ethnographique recense, enregistre (notes documentaires, phonogrammes, films cinéma ou vidéo) et analyse, avaient dans ce musée, selon le vœu de son fondateur Georges Henri Rivière, autant et parfois davantage de sens que les objets matériels eux-mêmes. Les techniques, avant le numérique, ne permettaient toutefois que faiblement de présenter au public, de manière aussi explicite que la culture matérielle, cette culture « immatérielle».

3 De fait, cette conception dont beaucoup d'aspects demeurent très modernes, a montré, avec le temps, son insuffisance, ce qui s'est traduit par une désaffection du public et une marginalisation des chercheurs. Le Ministère de la Culture et le CNRS ont donc été conduits à envisager une transformation radicale, qu'il n'est pas dans notre propos de décrire ici. On en trouvera les éléments essentiels dans le programme scientifique et culturel publié à son propos (Colardelle 2002). Cette transformation, pour faire court, concerne l'extension du champ géographique à l'Europe et à la Méditerranée, l'élargissement chronologique au dernier millénaire, voire davantage quand le besoin s'en fait sentir pour expliquer et montrer un fait culturel, la prise en compte des sociétés dans toutes leurs composantes et non seulement celle des classes populaires, et le recours à la transdisciplinarité, seule susceptible de permettre la compréhension de structures dont la complexité résiste à l'analyse unidisciplinaire. La décision a été prise de construire pour lui un nouveau bâtiment à Marseille, ville emblématique de la rencontre entre Europe et Méditerranée, du commerce et des contacts entre les cultures. Le futur établissement intègrera le prestigieux monument du Fort Saint-Jean, sur le Vieux Port, et disposera d'espaces mieux adaptés à son public comme à ses collections.

La forme muséographique donnée à l'origine au musée par Georges Henri Rivière, pourtant à l'époque innovante voire révolutionnaire, devra évoluer, à la fois pour prendre en compte ces transformations fondamentales et pour s'adapter aux goûts et aux techniques de communication actuels, sans renoncer à ses principes directeurs, toujours valables. La centralité de l'Homme, perdue de vue dans le projet initial, doit être retrouvée. Le concept de "galerie d'étude ", périmé parce que devenu trop partiel devant l'extension du champ patrimonial couvert (il y a vingt fois plus d'objets dans les réserves que dans la galerie d'étude, et certains secteurs de collection en sont complètement absents) doit laisser place à un centre documentaire multimédia, véritable «cybermusée » beaucoup plus nourri et plus commode d'accès, et à des « réserves visitables » dont le Centre du Conservatoire national des Arts et Métiers (CNAM) à SaintDenis a fourni une bonne application du principe.

5 Appliquées au domaine de la musique, ces caractéristiques, dans leur évolution, gardent toute leur valeur. Le MNATP, en principe, ne rend pas compte de la musique pour ellemême, mais comme l'un des traits culturels qui expriment les faits sociaux. Comme toujours, le concept de "populaire », en ce qu'il sépare trop radicalement son objet du domaine dit « savant », est discutable. Il s'agit au contraire d'appréhender la musique « de 
tous les jours " dans sa globalité, dans ses rapports avec la création savante, avec l'économie, avec les rythmes de la vie sociale et ses symboles. La musique et ses instruments se rapportent en effet à tous les aspects de la vie: le pouvoir, la fête, la religion, le travail. Les clarines sont des instruments de musique en même temps que des signes sonores de reconnaissance par le berger du troupeau dont il a la charge, et des supports de la littérature orale. La guitare électrique est à la fois un instrument de musique, un produit technique propre au siècle de l'électricité, le symbole de l'émergence de la jeunesse comme catégorie sociale et de la mondialisation culturelle, le signe de l'irruption de modèles héroïques nouveaux créés par les médias et le commerce, l'origine du problème de santé publique que le « décibélisme » engendre actuellement... La mise en perspective de la musique avec les autres faits sociaux autorise un autre regard, une autre analyse que la simple classification stylistique. Le propre de ce musée est de donner à voir et à apprécier cette relation dans sa complexité et dans son foisonnement de particularismes, mais aussi à en comprendre les ressorts fondamentaux dans leur universalité.

\section{L'ethnomusicologie de la France et les collections musicales du MNATP}

La musique est présente dès l'origine du Musée des Arts et Traditions populaires puisque les premières enquêtes de terrain remontent à 1939 (Gétreau 2004). D’abord matière scientifique, elle est pourtant recueillie dès cette époque de fondation pour devenir aussi matière muséographique.

7 C'est autour d'elle, qui se propose de garder mémoire et d'étudier les traditions musicales (du profane au sacré, du chant de travail à la musique de fête, de l'expression du peuple à celle du pouvoir), que s'est constitué à partir de la Seconde Guerre Mondiale, sous l'impulsion de Georges Henri Rivière et avec l'énergie de Claudie Marcel-Dubois et Maguy Pichonnet-Andral, un département d'ethnologie musicale, intitulé ensuite d'ethnomusicologie, et plus tard encore «Département de la musique et de la parole " (Cheyronnaud 2003).

Les missions furent [souvent organisées] avec l'appui du CNRS [...], elles furent toujours menées en équipe. Ces prospections ont systématiquement combiné l'étude du fait musical et celle du contexte sociologique, ethnographique et linguistique et ont toujours bénéficié du secours des techniques modernes. Chants, airs instrumentaux, cérémonies à éléments de musique et de danse, récitations modulées, formes vocales à la limite du langage et de la musique, signaux sonores aux frontières de la musique instrumentale, rythmes de travail et de jeux, informations parlées sur les traditions et les faits musicaux, sur la fabrication artisanale des instruments de musique furent rapportés de ces enquêtes (MarcelDubois 1960).

Les collections sont d'abord constituées d'enregistrements inédits réalisés par les ethnomusicologues et par l'ensemble des chercheurs du musée sur supports variés. Ils concernent la France métropolitaine mais aussi des pays d'ancien peuplement français et des pays francophones (Antilles, Louisiane, Québec, Ile de la Réunion et Ile Maurice, Belgique, Iles anglo-normandes, Suisse romande, Val d'Aoste, etc.). Des disques du commerce (phonogrammes édités) forment également une section au sein de laquelle on dénombre un ensemble entré sous le nom de « Musée de la chanson ». 
L'ensemble des fonds sonores se compose de 1507 collections (ou sources) comportant en tout 78000 items, qui se décomposent en 44000 phonogrammes inédits et environ 34000 phonogrammes édités. Le Musée de la chanson représente parmi ces derniers 1857 phonogrammes.

10 Ayant soumissionné lors de l'appel à projet de la Mission de la Recherche en 1999, le MNATP a bénéficié, comme quatre autres centres d'archives sonores français, du plan national de numérisation du Ministère de la Culture. Plus de 1400 heures d'enquêtes inédites sont dès à présent sauvegardées sur supports CD (copie master), la mise à disposition sur place d'une copie de consultation permettant dorénavant une diffusion très aisée de ces collections sonores.

11 Du côté des collections matérielles, la collecte des objets représentatifs des pratiques musicales populaires ne s'est faite qu'au détour des enquêtes, productives de milliers de phonogrammes, mais d'un nombre d'objets relativement réduit. Cependant, par rapport aux collections publiques musicales en France, le MNATP dispose de l'ensemble d'instruments traditionnels français le plus développé (environ 1500). Il conserve aussi de l'outillage et des séquences de facture d'instruments souvent spécialement constituées dans des ateliers traditionnels. Il dispose d'un atelier de graveur de musique sans équivalent. Mais il a aussi une collection très développée de musique imprimée (notamment de chansons françaises remontant au siècle dernier) dont la majorité date de la première moitié du XXe siècle (6500 partitions), ainsi que des souvenirs de chanteurs. C'est ce qui constitue, avec les disques mentionnés plus haut, le « Musée de la Chanson ", lancé en 1962 à partir d'un appel radiophonique de Louis Merlin et de Georges Henri Rivière, qui était lui-même un musicien averti et un passionné de chanson.

Dans d'autres départements du musée, on trouvera aussi des documents d'archives concernant la musique (carnets de chanteurs par exemple), un intéressant répertoire de colportage (recueils de chansons et cantiques remontant au XVIII siècle) (Gétreau 2002), des collections d'iconographie musicale variées pour le XIX ${ }^{\mathrm{e}}$ siècle (principalement estampes, mais aussi tableaux, céramiques musicales).

13 L'analyse numérique des acquisitions d'instruments de musique (en ne comptant que pour une unité chaque ensemble d'outils lorsqu'il s'agit d'un atelier de fabrication) fait apparaitre de grandes variations suivant les périodes, effet du dynamisme ou de la régression du département concerné ou du musée tout entier :

\begin{tabular}{|l|l|}
\hline $1881-1935$ & 18 (anciens fonds du Musée de l'Homme) \\
$1936-1949$ & 66 \\
$1950-1959$ & 433 \\
$1960-1969$ & 519 \\
$1970-1979$ & 85 \\
$1980-1989$ & 111 \\
$1990-1995$ & 0 \\
$1995-2002$ & 155 \\
\hline
\end{tabular}

14 On voit donc bien que ce sont les décennies 50 et 60 , et plus précisément les années 1952-1969, qui manifestent la plus grande activité. La chute date de 1979 et, à deux années favorables près (1985 et 1986), il faut attendre 1995, après cinq années totalement 
blanches, pour voir relancer les acquisitions, avec des œuvres de qualité sinon très nombreuses.

Sur le plan qualitatif, les collections reflètent l'orientation classique du musée aujourd'hui remise en cause, comme on l'a vu - vers le monde rural traditionnel, à quelques rares exceptions près (orgues de manège, instruments de cirque, quelques instruments de jazz-bands et guitares électriques). Les points forts sont sans conteste les séquences de fabrications (tambourins du Var, vielles à roue de Jenzat, épinettes du Val d'Ajol, cornemuses du Centre, accordéons parisiens), et les objets accompagnés d'une enquête ethnographique (vallée d'Ossau, Aubrac, instruments de Carnaval de Vence, etc.). A cela s'ajoutent 6500 partitions, en particulier les « petits formats » entre 1825 et 1950.

Depuis 1995, la politique d'acquisition cherche, à partir de l'analyse du fonds existant, à combler les lacunes chronologiques et typologiques, en privilégiant des instruments bien référencés dont on connaît la fonction, l'utilisation et les conditions de jeu. L'instrumentarium de plusieurs musiciens de bals auvergnats de Paris a ainsi été acquis récemment. La préparation de l'exposition Musiciens des rues de Paris a également été l'occasion d'une série de découvertes intéressantes, tels le tableau intitulé L'aveugle Frélon d'Antoine-Pierre Mongin (1814) ou les parures d'hommes-orchestres de la famille Vermandel. De même l'exposition Souffler c'est jouer (Gétreau et Montbel 1999) a permis, grâce aux contacts d'Eric Montbel, d'élargir la représentativité du corpus de cornemuses à différents modèles de chabretas limousines. L'accent est mis sur l'acquisition d'œuvres dotées d'un contexte, choisies pour la représentativité sociale et culturelle de celui-ci tout autant que pour leur valeur intrinsèque au titre de l'organologie ou de l'esthétique.

Fig. 1 : La famille Vermandel dans les rues de Paris, vers 1977. MNATP, cliché anonyme

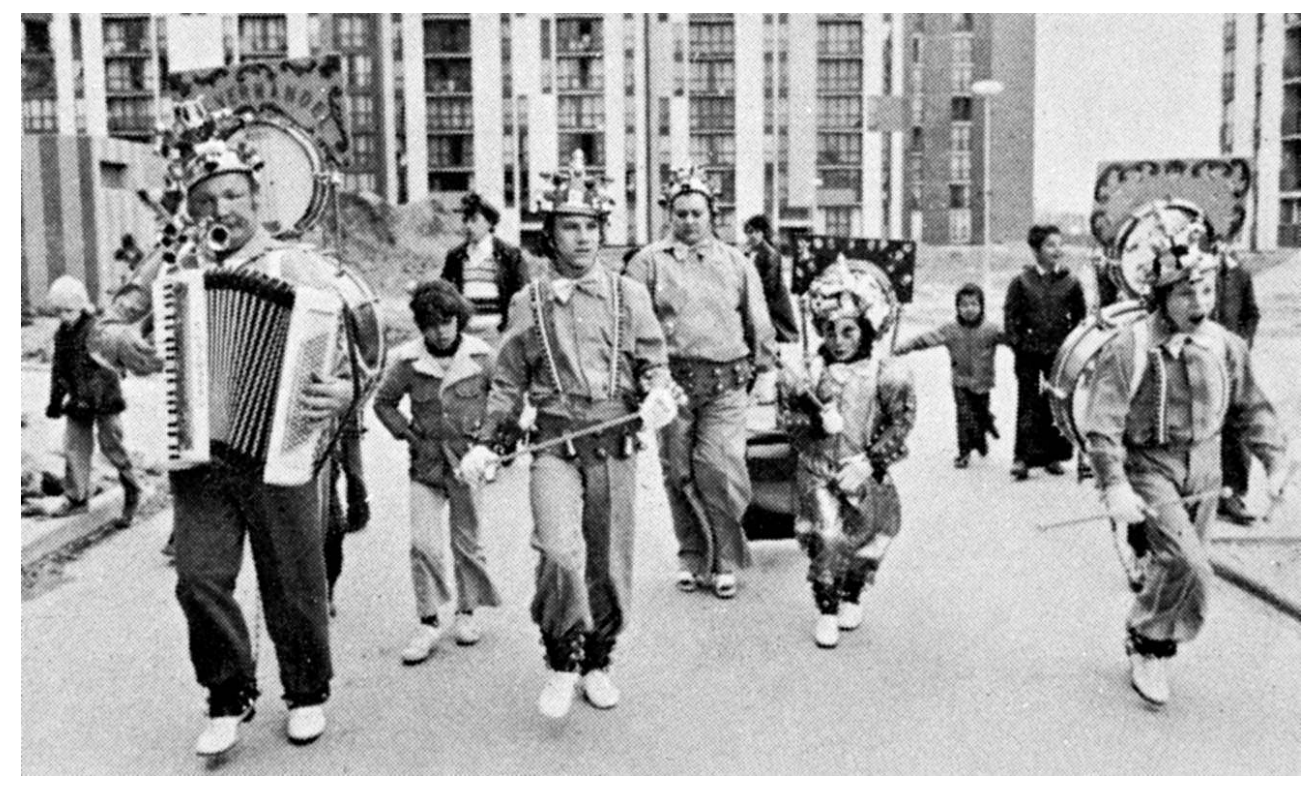

La lacune principale des collections a trait, dans ce domaine comme dans les autres, à l'après-guerre. Cette période est très insuffisamment représentée, ne rendant absolument pas compte des phénomènes musicaux populaires des années 60 à aujourd'hui, rock et musique amplifiée par exemple. Ces derniers mois, grâce aux travaux de Marc Touché, sociologue au CEF, sur les musiques actuelles amplifiées, le musée s'est enrichi des Archives du Golf Drouot, du matériel de plusieurs groupes et d'une console utilisée par les 
Pink Floyd, les temps contemporains étant désormais clairement inscrits au cœur de nos préoccupations.

\section{La musique dans la Galerie d'étude et la Galerie culturelle du MNATP}

Si, pendant trente ans, les travaux ethnomusicologiques du musée n'ont été restitués que dans le cadre restreint d'expositions temporaires non spécialisées, on peut se demander "Quelles étaient les lignes de force qu'une muséologie inventive comme celle de Georges Henri Rivière devait exploiter à partir des acquis du département d'ethnomusicologie ATP ? Le principe de cette muséologie moderne appliquée à l'ethnomusicologie dans une institution où tout était à construire, a été fondé pour l'essentiel sur une association aussi étroite que rigoureuse entre les activités de la recherche et de la conservation, les premières, en règle générale, alimentant les secondes et les secondes soutenant ou parfois même déterminant les premières. C'est grâce à ce va-et-vient constant de recherches et de collectes sur le terrain, d'études érudites et de réalisations techniques et muséographiques que les collections peuvent et purent être constituées, identifiées, documentées, analysées, enrichies, conservées, restaurées, exposées » (Marcel-Dubois 1989 : 183).

Fig. 2 : Georges-Henri Rivière en 1982 devant l'une des vitrines consacrées à la musique dans la Galerie culturelle du MNATP

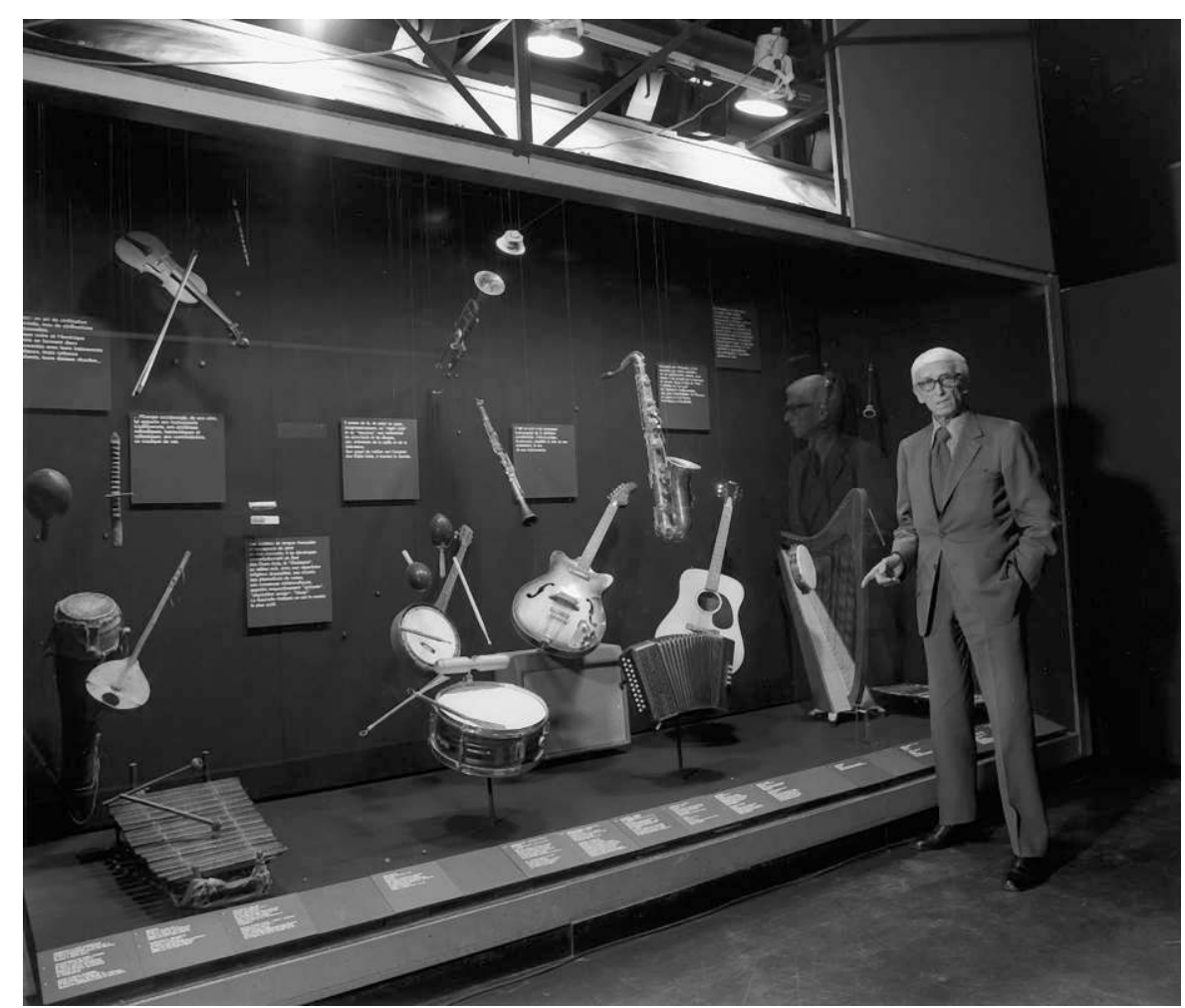

Photo : André Pelle

La Galerie d'étude, ouverte au public en 1972 présenta, jusqu'à sa fermeture en 1996, 319 instruments et objets. Claudie Marcel-Dubois et Maguy Pichonnet-Andral, dans le catalogue descriptif qu'elles préparèrent, indiquaient que son programme cherchait « par 
divers angles d'approche, à éclairer certains des problèmes posés par les musiques traditionnelles françaises et exprimables à travers les instruments de musique. C'est ainsi que cinq thèmes majeurs se partagent l'espace de la vitrine : la partie la plus étendue est réservée à la typologie des instruments de musique. Etablie à partir des normes classificatoires internationalement adoptées, cette systématique démontre que le matériel instrumental français couvre pratiquement toutes les catégories organologiques. Les quatre autres thèmes sont consacrés successivement : à la facture instrumentale, aux modalités d'exécution de la musique instrumentale, à la représentativité de l'instrument de musique considéré comme emblème régional, à la représentativité de l'instrument de musique considéré comme emblème social » (Marcel-Dubois et Pichonnet-Andral 1988).

De son côté, la Galerie culturelle présente, depuis son inauguration en 1975, une centaine d'instruments de musique et quelques objets qui en renforcent le contexte, lesquels ont été sélectionnés en vue de privilégier les usages sociaux de la musique (communication, techniques, rituels, art). Comme l'indique le Petit guide (Goffre 1991), la vitrine Musique et société «a adopté le plan général de la Galerie culturelle et montré la place des phénomènes sonores dans les accomplissements humains saisis successivement à travers l'histoire, les activités techniques, les coutumes et les croyances, les institutions et les œuvres ». Rivière avait ainsi demandé que cette vitrine rapproche trois catégories de documents considérés en fait comme "œuvres» (images, objets, sons), selon trois registres de lecture. La lecture en paraît aujourd'hui extrêmement structuraliste, très dense et sans doute infiniment loin d'une nécessaire approche sensible. Elle n'échappe pas non plus à une appréhension des phénomènes musicaux « révolus », ignorante qu'elle est des pratiques observées depuis un demi siècle.

On conçoit, en conséquence, combien les musiques «populaires » doivent prendre, dans le futur établissement, une place plus large, à la mesure du nouvel éventail que permet la reprise des acquisitions et des enquêtes. Il est un peu tôt pour donner les grandes lignes d'un projet qui est en cours d'élaboration ; mais on peut d'ores et déjà dire que la musique elle-même - et pas seulement les instruments - sera davantage présente, et que l'élargissement de la thématique du musée à la ville et au contemporain va la mêler à des problématiques culturelles diversifiées à la mesure de l'élargissement des frontières et des civilisations qu'il va évoquer.

\section{Comment définir la fonction singulière du MUCEM parmi les autres institutions culturelles s'occupant de musique?}

Le musée, s'il traite de la musique, peut être - mais ce n'est pas une constante - un conservatoire d'archives sonores. Or d'autres institutions partagent avec lui cette fonction : les radios, les bibliothèques, avec en tête la Bibliothèque nationale de France, les archives départementales, les centres de recherche, les associations de musique et danse traditionnelle notamment (Loddo, Bouthillier 2000); le musée a sans doute un atout, celui d'avoir aussi des archives graphiques (photographies, films, sources écrites) lesquelles accompagnent rarement les fonds des autres archives sonores.

Le musée, lorsqu'il a des moyens de programmation et de production, est un lieu de diffusion, de concert et de spectacle. Mais les festivals et les salles spécialisées ont une place prépondérance pour la diffusion de ces musiques et ont joué un rôle culturel 
incomparable. Une politique discographique a toujours manqué au MNATP, dont l'éditeur institutionnel, la Réunion des Musées Nationaux, n'est pas outillée pour la musique. Saurons-nous trouver des solutions pour l'avenir en nous appuyant sur l'expérience d'autres institutions?

Le musée comme foyer de recherche et lieu d'enseignement : il partage ce rôle avec des laboratoires à part entière, avec des universités et avec des associations. Dans le cas du MNATP, on voit bien que nombre d'initiatives, à Paris comme sur le territoire, se sont construites en opposition aux tendances qu'il avait développées. Le laboratoire intégré au musée n'a plus de chercheur ethnomusicologue CNRS depuis une décennie. Les travaux de sociologie (notamment le champ des musiques amplifiées porté par Marc Touché) et ceux d'organologie (que nous développons) ne permettent pas une approche globale. Le cursus de l'Ecole du Louvre en Anthropologie sociale et culturelle de l'Europe que nous avons récemment initié propose une toute première approche de l'ethnomusicologie et l'encadrement de travaux de premier cycle et de muséologie où le musical est loin d'être absent. Mais on voit bien toute l'utilité qu'il y aurait à nouer des conventions de travail avec l'enseignement universitaire spécialisé, dans la tradition du Département d'ethnomusicologie du Musée de l'Homme, au bénéfice des étudiants comme de l'institution. Le temps est à la coopération.

NLe musée comme collection d'objets matériels à deux et trois dimensions : là encore il n'en a pas l'apanage. De nombreuses bibliothèques ont la charge de fonds musicaux et iconographiques comparables. Ses quelques centaines de recueils de colportage de chansons et cantiques sont à mettre en regard des milliers qui sont conservés au Département de la musique de la BNF (fonds Coirault et Weckerlin) et ses très nombreuses partitions imprimées de chansons sont à considérer face au dépôt légal exhaustif de la même institution. Ses 1400 estampes à sujet musical sont à mettre en rapport avec les centaines d'œuvres similaires conservées au Cabinet des Estampes et de la Photographie de la BNF, au cabinet d'art graphique du Musée Carnavalet et dans les mille musées en région; ses vêtements et accessoires de musiciens et de vedettes du Music Hall ne sont pas non plus uniques si l'on songe au Département des arts et spectacles de la BNF ou au Musée de Montmartre. C'est sans doute dans le domaine des instruments de musique, mais avec des forces et des faiblesses (quasi absence d'instruments de la deuxième moitié du XXe siècle; absence pour l'instant d'instruments européens et méditerranéens - les fonds du Musée de l'Homme et des dépôts du Musée $\mathrm{du}$ Quai Branly viendront partiellement combler cette lacune -, trop faible représentativité des instruments mécaniques et électriques), que l'on touche à l'une des missions fondamentales du musée. Parmi les collections publiques instrumentales, ses quelque 1500 items, auxquels s'ajoutent ses ateliers et outillages de facteurs et de graveurs de musique, lui donnent encore une relative prépondérance dans le domaine des instruments populaires du domaine français; mais le travail de collecte entrepris au musée de Montluçon depuis dix ans relativise largement cette position, tandis que le Pôle de l'Accordéon de Tulle est très ouvert sur l'Europe, la naissance, bien que difficile, du musée de Mirecourt montrant qu'une dynamique est en cours à l'échelle de tout le territoire. Et c'est peu dire des réalisations récentes en Europe, comme par exemple au Musée des Instruments de musique de Bruxelles qui consacre une large section aux pratiques populaires.

Tout bien considéré, et prenant en compte ce partage de fonctions avec d'autres institutions, cette absence de prédominance et de représentativité dans le domaine des collections matérielles, sachant que leur perception par le public et les 
chercheurs pose question, on voit bien que le futur musée doit construire sa vocation autrement. Il le peut probablement dans le domaine des expositions, la mise en espace des objets de la musique et la représentation de la matière musicale. Nous reviendrons, non sur les multiples expériences présentées ici où là, mais sur quelques orientations tant dans le domaine du "permanent»- nous préférons aujourd'hui parler d'espaces de référence, évolutifs et adaptables - que de l'événementiel. voie de disparition; musiques de communautés et musiques d'artistes, quels choix proposer au musée ? Loin du village et de sa tradition in vivo en marche, le musée doit-il être, dans le contexte urbain qui est le sien, l'un des rares "conservatoires" de «traditions-sources»? Sur quels critères définir l'espace des musiques traditionnelles? Où établir les frontières musicales de l'Europe et de la Méditerranée ? Le musée a-t-il un rôle à jouer pour rassembler un «Atlas" des musiques européennes? Au plan sonore, cela a-t-il encore un sens à l'ère de la numérisation, des réseaux multimédia et de l'Internet? Au plan des collections matérielles, faut-il que les collections euroméditerranéennes d'instruments quittent les réserves du Quai Branly? Au-delà d'une sélection limitée visible en permanence, le fonds sera-t-il plus « utile » à la communauté à Paris où à Marseille ? Concernant le Maghreb - mais c'est un exemple parmi bien d'autres -, la prise en compte de ses musiques se fera-t-elle en une programmation concertée entre les deux établissements (celle des thématiques de référence, des activités, des collectes) ? En terme de diachronie, quand commencer? En synchronie, où s'arrêter?

De même si le musée est le miroir où une population se retrouve pour renvoyer son image aux autres, les musiques amplifiées qui, selon la formulation de Véronique Mortaigne, 
ressortissent d'une "sorte de tribalisme », ont tout autant droit de cité au futur musée. Immergé dans la floraison des musiques hybrides, conscient du nivellement planétaire, des usages boulimiques de timbres exotiques - le zapping fait que l'instrument traditionnel peut être réduit au rôle d'alibi par un collage où il perd jusqu'à sa consistance technique - le public, si volatile et multiple soit-il, doit pouvoir trouver au musée un lieu de rencontre entre des tendances en apparence opposées, un lieu où le nomadisme musical marque un temps d'arrêt, un temps de réflexion et de prise de conscience, une occasion de partager une autre expérience.

\section{Son et musique dans les espaces et les activités du futur musée}

Le "son comme environnement sonore »: on aimerait qu'il soit partie intégrante de la syntaxe du futur musée. Que chaque thématique d'exposition s'exprime aussi par le bruit naturel, le bruit technique, le bruit symbolique.

Que le «son comme parole» soit abondant et que l'on puisse, autant que possible, voir parler notre interlocuteur pour éviter l'abstraction des voix sans visage. On observera qu'au delà d'un discours « signalétique » qui ne passe pas uniquement pas le cartel écrit, c'est l'association du témoignage qui est en jeu dans le parcours muséographique. Que la question centrale des langues de l'Europe est un sujet en soi, mais que des éléments du parcours pourraient être systématiquement désignés par « les mots pour le dire » dans les différentes langues convoquées. La littérature orale n'a jamais eu droit de cité dans les galeries du MNATP depuis qu'à la fin des années 70, les cabines d'écoute sont tombées en panne. Un espace particulièrement étudié, ayant une certaine intimité et une poésie suggestive, pourrait être dévolu à l'écoute en petits groupes.

Fig. 3 : Vue du Fort Saint Jean à Marseille, site du futur Musée des Civilisations de l'Europe et de la Méditerranée. Photo : Michel Colardelle, 2002

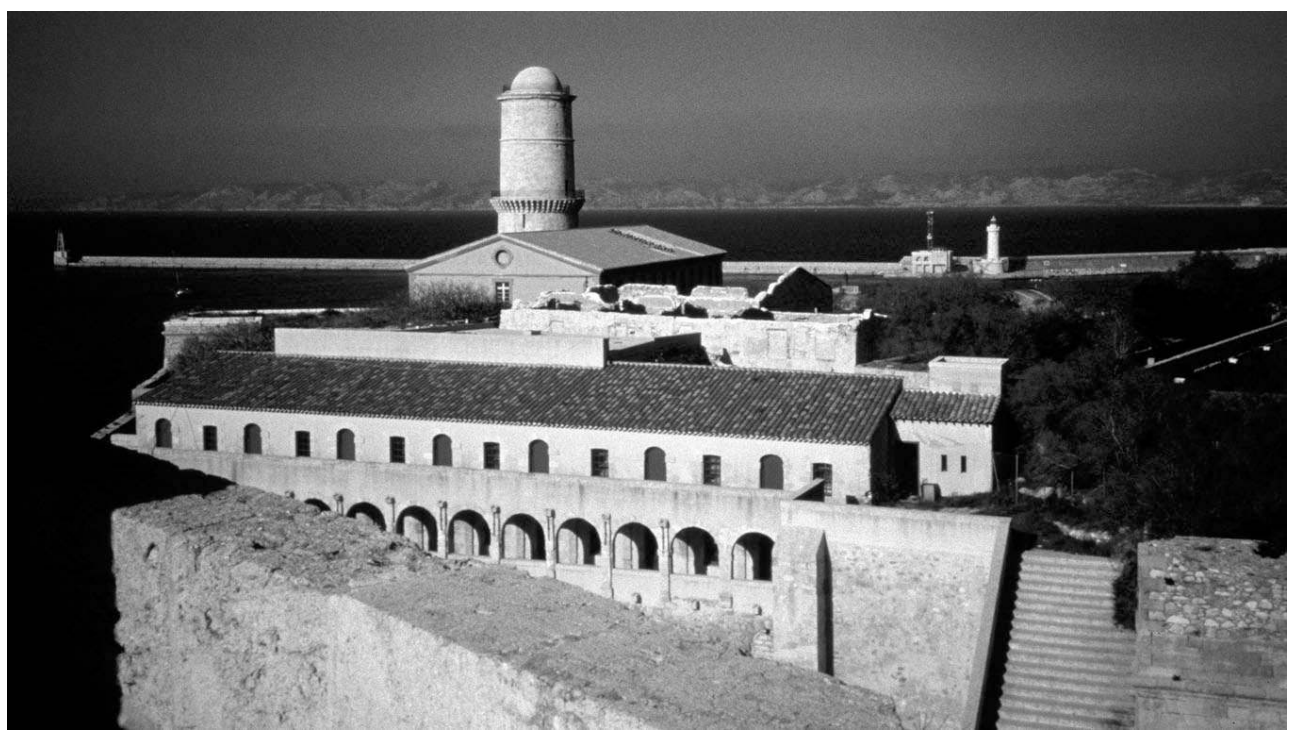

Quant au «son musical», on aimerait que le visiteur puisse soit le partager, soit l'intérioriser, soit l'analyser, soit le produire. Cela implique des dispositifs chaque fois 
différents, et non un système unique sur l'ensemble du parcours. Un câblage général des salles a donc été préconisé dès la phase de concours architectural.

Les lieux collectifs d'écoute devraient, là encore, répondre parfois à des espaces isolés acoustiquement (telles des petites salles à la jauge d'un groupe scolaire), soit de petites salles semi ouvertes, intégrées aux cinq modules des expositions de référence. On aimerait qu'elles s'inspirent de la définition que Bernard Lortat-Jacob en donnait dans son texte de 1999 "Du côté du spectacle, ou comment améliorer les conditions de diffusion des musiques du monde? ? (p. 166). S'ajoutent aussi la chapelle romane de deux cent places du Fort Saint-Jean, le grand amphithéâtre du musée, le café musical, et le théâtre de plein air, ces derniers espaces devant permettre à la danse de s'improviser avec le public.

L'écoute et l'étude des fonds sonores numérisés se fera à la médiathèque. Rassemblant tous les supports (imprimés, archives, photographies, dossiers, images et films numérisés, etc.) en un lieu unique de consultation, elle comportera soixante-cinq postes multimédia.

\section{La musique dans le projet muséographique global} les règles du savoir-vivre, le développement de la connaissance scientifique, les idées et les usages religieux, les formes de l'habitat, les modes de préparation des aliments, les systèmes d'organisation sociale ;

1. créer un lieu de ressourcement;

2. inciter le public à la distanciation, l'appréciation et la réflexion ;

3. contribuer à une prise de conscience du rôle de chaque groupe ou de chaque individu dans la production de modèles collectifs ;

4. apporter au visiteur des connaissances dans le respect des siennes pour créer un dialogue ;

5. solliciter le jugement critique qui définit le musée citoyen ;

6. proposer une muséographie fondée sur l'étonnement ;

7. tenir compte de la nature des différents publics;

8. élaborer une complémentarité entre exposition de référence et exposition temporaire ;

9. faire un musée convivial, où l'on peut vivre une expérience originale : allier plaisir et connaissance.

Parmi les principes généraux de la muséographie, notons qu'ils veulent:

1. exprimer la diversité des cultures par une démarche comparative à travers des objets témoins: parentés, apparentements, différences/mécanismes de la différenciation, confrontations et conflits ;

2. faire appel aux cinq sens ;

3. éviter l'exhaustivité mais privilégier la représentativité ;

4. choisir des thématiques qui soient mieux adaptées à l'expression muséographique qu'à celle du cinéma, du théâtre ou du livre, pour s'inscrire dans la complémentarité des autres institutions culturelles ;

5. éclater chaque problématique traitée en pôles physiquement distants; les séparer par des cheminements qui donnent un temps au repos, à la méditation, à la réflexion et au dialogue. 
Ces parcours doivent être largement ouverts sur le paysage : la mer, la ville, le port, les jardins ;

6. laisser au visiteur le choix de l'ordre du cheminement. figures musicales du Paradis sont multiples, qu'elles soient d'inspiration sacrée (telles les lamentations), qu'elles soient militantes et porteuses d'un idéal ou qu'elles évoquent par exemple les paradis artificiels, comme ceux du rock'n roll des années soixante dans les jardins londoniens. L'eau peut être celle des orgues hydrauliques, des jeux de bols et des vases sonores mais aussi celle des fêtes nautiques et des chants de lavandières. La cité est celle des sonneurs de tours, celle des bals populaires ou des marques du pouvoir, celle des musiciens de rue et des bandes ambulantes, mais aussi celle des « cités » contemporaines où se développe le rap. La circulation peut être entendue comme celle des hommes, musiciens ambulants, musiciens migrants, musiciens exilés; celle des supports (les recueils de colportage, les instruments); celle des formes (emprunts d'un cercle social à un autre, du religieux au profane, d'une rive à l'autre). Les routes de la foi ne peuvent se passer de la musique qui marqua les parcours et les stations. En Méditerranée, c'est celle des musiques arabo-andalouses et de leur diffusion dans toutes les couches de la société musicale occidentale à la fin du Moyen Age. Le luth et le clavecin des aristocraties européennes en sont les descendants, comme la guitare électrique et le synthétiseur. Masculin/féminin, ce sont les voix de berceuses et les plaintes funèbres des femmes, le hautbois comme monopole masculin, mais aussi les quatre voix d'hommes d'où se dégage la quintina, voix féminine fusionnelle de l'accord parfait évoquant, comme le remarque Bernard Lortat-Jacob, «la Vierge, la mère du Christ, la femme, la fiancée, sa propre mère ».

41 Ces thèmes seront en relation avec une programmation d'expositions temporaires dont le champ est illimité et qui peuvent être le point d'aboutissement de recherches ethnomusicologiques. Mais ces thèmes de référence sont par ailleurs accompagnés de «salles de cartes» sur la linguistique, l'histoire, la géographie, les sites, les grands hommes. C'est peut-être là qu'il faut un espace particulier autour de la diversité des voix de l'Europe et de la Méditerranée. A moins que, rompant avec les typologies intellectuelles et souvent stériles des musées traditionnels, on ne propose une présentation instrumentale foisonnante des «modes de jeu» : frapper, secouer, racler, frotter, souffler. On voit bien que les suggestions peuvent être infinies. Reste à les formaliser, à trouver un équilibre et un consensus entre les attentes des experts et celles du public. La mise en place d'un groupe de propositions semble maintenant indispensable pour répondre aux contraintes de la programmation. Il faudrait pouvoir en définir le fonctionnement et la composition. Par ailleurs des collaborations sont établies au plan des politiques d'acquisition et, récemment, pour la préparation d'un outil commun au

Cahiers d'ethnomusicologie, 16 | 2011 
Musée de la Musique, à celui du quai Branly et au MNATP : un thesaurus des instruments de musique destiné aux chantiers des collections en cours ou à venir.

Pourquoi ne pas conclure par le musée musical idéal de Georges Henri Rivière ; non celui qu'il a réalisé et ouvert au bois de Boulogne en 1972, l'année où Alan Stivell se produisait pour la première fois à l'olympia, mais plutôt ce musée de civilisation musicale qu'il rêvait pour La Villette et qui reste encore à naître :

Temple des muses à sa manière, Mouseion, le musée, dès longtemps, accueille la musique. La musique, parmi les hommes, est partout. Dans la nuit de leur temps, hûchement, avant la parole. Tour à tour muse d'Apollon ou de Caveau; charivari de cocu ou fugue de Bach ; maîtrise de cathédrale ou fest-noz ; vingt-quatre violons ou bal parquet; cri de métier ou vocalise de reine de la nuit; tambour de shaman, extasiant, de bataille, meurtrier, à escorter la mort, voilé; temps des cerises ou slogan de manifestation ouvrière ; guimbarde ou synthétiseur [...] $]^{\text {. }}$

Puissions-nous en garder quelque inspiration.

\section{BIBLIOGRAPHIE}

COLARDELLE Michel, Colette FOISSEY et Edouard de LAUBRIE, dir., 1999, Réinventer un musée. Le musée national des Arts et Traditions populaires/Centre d'Ethnologie Française. Paris : Ecole du Louvre.

COLARDELLE Michel, dir., 2002, Réinventer un musée. Le musée des Civilisations de l'Europe et de la Méditerranée à Marseille. Paris : Réunion des musées nationaux.

CHEYRONNAUD Jacques, 1986, Mémoires en recueils. Jalons pour une histoire des collectes musicales en terrain français. Montpellier : ODAC.

CHEYRONNAUD Jacques, 2003, «Ethnographie musicale française (1934-1945). Sur une chronologie fondatrice ", in Jacques Cheyronnaud : Musique, politique, religion. De quelques menus objets de culture. Paris : L'Harmattan : 157-197.

GETREAU Florence, 2002, «La chanson de colportage éditée hors de Troyes dans les collections du Musée national des Arts et Traditions populaires : une esquisse », in Marie-Dominique Leclerc et Alain Robert, dir. : Chansons de colportage. Reims : Presses universitaires : 78-91.

GETREAU Florence, 2004, « Recherche et maintien de la tradition musicale populaire en France : positions de principe, méthodes d'observation et réalisation du MNATP », in Colloque international : Du folklore à l'ethnologie. Institution, musées, idées en France et en Europe de 1936 à 1945. Paris : MNATP, 19-21 mars 2003, à paraître.

GETREAU Florence, dir., 1997, Musiciens des rues de Paris. Catalogue de l'exposition, Paris, Musée national des Arts et Traditions populaires, Réunion des Musées Nationaux.

GETREAU Florence et Eliane DAPHY, dir., 1999, Musiques dans la rue. Ethnologie française 1999/1 : 8-65.

GETREAU Florence et Eric MONTBEL, dir., 1999, Souffler, c'est jouer : chabretaires et cornemuses à miroirs en Limousin. Catalogue de l'exposition, Paris : Musée national des Arts et Traditions populaires ; Saint-Yrieix-la-Perche : Salle Attane ; Saint-Jouin-de-Milly : Editions Modal/Famdt. 
GOFFRE Annie, 1991, Paris. Musée national des Arts et Traditions populaires. Petit guide. Musique. Galerie culturelle. Paris : Réunion des musées nationaux.

LODDO Daniel et Robert BOUTHILLIER, 2000, Les archives sonores en France. Saint-Jouin-de-Milly : Editions Modal.

LORTAT-JACOB Bernard, 1999, « Derrière la scène », in Laure Bernard, Philippe Gouttes et Chérif Khaznadar, dir. : Les musiques du monde en question. Internationale de l'Imaginaire. Paris : Babel :

156-171.

MARCEL-DUBOIS Claudie, 1960, « Ethnomusicologie de la France. 1945-1959 », Acta Musicologica :

113-121.

MARCEL-DUBOIS Claudie, 1972, « Phonothèque du musée national des Arts et Traditions populaires ", IASA Phonographic Bulletin $4: 23-26$.

MARCEL-DUBOIS Claudie, 1989, « Visite au département d'ethnomusicologie du musée des Arts et Traditions populaires ", in La muséologie selon Georges Henri Rivière. Paris : Dunod : 183-184.

MARCEL-DUBOIS Claudie et Maguy PICHONNET-ANDRAL, 1988, Galerie d'étude. Section « Musique ». Catalogue des instruments présentés. Manuscrit dactylographié, 47 p.

\section{NOTES}

1. Allocution prononcée par G.H. Rivière, le 25 septembre 1975, à la mémoire de $\mathrm{M}^{\text {me }}$ Hubert Pelletier de Chambure, née Geneviève Thibault.

\section{RÉSUMÉS}

La musique est présente dès l'origine du Musée des Arts et Traditions populaires puisque les premières enquêtes de terrain remontent à 1939. Enquêtes, définition d'une ethnomusicologie de la France, constitution d'une phonothèque et de collections instrumentales, expositions thématiques permanentes, autant de réalisations de l'équipe fondatrice. Ces dernières années d'autres formes de collecte (notamment sur les pratiques contemporaines) et de muséographie ont été expérimentées, tandis que la sauvegarde les collections sonores a accompagné l'ouverture de l'institution aux acteurs de tous milieux. La préparation du Musée des Civilisations de l'Europe et de la Méditerranée propose des perspectives inédites tant au plan des collaborations que de la prise en compte du public. Michel Colardelle en trace les enjeux intellectuels et culturels, tandis que Florence Gétreau s'interroge sur la présence du son et de la musique dans la future institution. 


\section{AUTEURS}

\section{FLORENCE GÉTREAU}

Florence Gétreau, conservateur au Musée instrumental du Conservatoire de Paris puis chef de projet du musée de la Musique, est chargée du département de la musique et de la parole du Musée national des Arts et Traditions populaire depuis 1994. Elle enseigne l'organologie et l'iconographie musicale au Conservatoire de Paris. Chercheur à l'Institut de Recherche sur le patrimoine musical en France (UMR 200 du CNRS), elle y a créé en 1995 la revue scientifique annuelle Musique-Images-Instruments. Elle a été lauréate du Curt Sachs Award en 2002.

\section{MICHEL COLARDELLE}

Michel Colardelle, conservateur général du patrimoine, est directeur du Musée national des Arts et Traditions populaires et du Centre d'ethnologie française (UMR 306 du CNRS) depuis 1996. Fondateur et directeur du Centre d'Archéologie Historique des Musées de Grenoble et de l'Isère (1976-1983), conseiller technique chargé des Musées et des Arts plastiques (1984-1986) puis conseiller technique chargé du Patrimoine et des Archives au Cabinet du Ministère de la Culture (1988-1989), il est directeur de la Caisse nationale des Monuments historiques et des sites (1989-1991) puis directeur de Cabinet du Ministère de la Jeunesse et des Sports (1991-1993). Chargé de mission auprès du Directeur des Musées de France (1993-1996), il rejoint le MNATP en 1996. Il est directeur du projet de Musée des Civilisations de l'Europe et de la Méditerranée à Marseille. Spécialiste d'archéologie médiévale, il est l'auteur de nombreuses publications et catalogues d'expositions. Il est professeur à l'Ecole du Louvre. 